\title{
ON THE GLOBAL DIMENSION OF CERTAIN PRIMITIVE FACTORS OF THE ENVELOPING ALGEBRA OF A SEMI-SIMPLE LIE ALGEBRA
}

\author{
TIMOTHY J. HODGES AND S. PAUL SMITH
}

\section{Introduction}

Let $\mathfrak{g}$ be a finite-dimensional semi-simple Lie algebra over an algebraically closed field $k$ of characteristic zero and let $\mathfrak{g}=\mathfrak{n}^{+}+\mathfrak{h}+\mathfrak{n}^{-}$be a triangular decomposition of $\mathfrak{g}$. Denote by $U=U(\mathfrak{g})$ the enveloping algebra of $\mathfrak{g}$. For $\lambda \in \mathfrak{h}^{*}$, denote by $D_{\lambda}$ the primitive factor ring $U / \mathrm{ann}_{U} M(\lambda)$, where $M(\lambda)$ is the Verma module of highest weight $\lambda-\rho$ (where $\rho$ is the half-sum of the positive roots). The main aim of this paper is to prove Theorem 3.9, which states that if $\lambda$ is regular, then $\operatorname{gldim} D_{\lambda} \leqslant \operatorname{dim}_{k} \mathfrak{n}^{+}+n(\lambda)$, where $n(\lambda)$ is a non-negative integer less than or equal to $\operatorname{dim}_{k} n^{+}$.

In [12] Levasseur computed the injective dimension of $D_{\lambda}$ in terms of the Gelfand-Kirillov dimension of $L(\lambda)$, the unique simple quotient of $M(\lambda)$. For $\lambda$ regular, Theorem 3.9 implies that the global dimension of $D_{\lambda}$ is finite and hence must be equal to the injective dimension of $D_{\lambda}$. It can be shown that this figure coincides with the bound given in Theorem 3.9 (the authors would like to thank Levasseur for pointing this out). On the other hand, if $\lambda$ is not regular, Joseph and Stafford [11] have shown that gldim $D_{\lambda}=\infty$.

Special cases of this result have already appeared in the literature. In particular Stafford [17] computed the global dimension of $D_{\lambda}$ for all $\lambda \in \mathfrak{h}^{*}$ in the case when $\mathfrak{g}=\operatorname{sl}(2, \mathbb{C})$. On the other hand Roos [15] computed gldim $D_{\lambda}$ for general $g$ when $\lambda$ satisfies some transcendental but generic conditions.

This paper is divided into two distinct parts. In Section 2 we prove a result analogous to a theorem of Roos relating the weak global dimension of a ring $R$ to that of certain sets of torsion-theoretic localisations. If $R$ is a commutative ring and $\left\{S_{i}\right\}_{i \in I}$ is a set of localisations of $R$ such that $\oplus S_{i}$ is faithfully flat as an $R$-module, then it is well known that wgldim $R=\sup \left\{\right.$ wgldim $\left.S_{i}\right\}$. To what extent this result is true for non-commutative rings is unknown. Roos and others have proved various results under the assumption that wgldim $R$ is finite (see for instance $[3,7,13,14]$ ). We prove here a slightly more general sort of result than Roos's which does not require this assumption. However, it is necessary to impose certain additional assumptions on the localisations $S_{i}$. The special case of this result needed for the second part is the following. Let $R$ be a prime Noetherian ring and let $S_{i}, i=1, \ldots, n$ be a finite collection of rings lying between $R$ and its quotient ring. If $\oplus S_{i}$ is a faithfully flat right $R$-module and $S_{i} \otimes_{R} S_{j} \cong S_{j} \otimes_{R} S_{i}$ as $R$-R-bimodules for all pairs $(i, j)$, then

$$
\text { gldim } R=\operatorname{wgldim} R \leqslant \sup \left\{\operatorname{wgldim} S_{i}+\operatorname{lfd}_{R}\left(S_{i}\right)\right\}
$$

(where $\operatorname{lfd}_{R}\left(S_{i}\right)$ denotes the flat dimension of $S_{i}$ as a left $R$-module). An example is given where the theorem fails if the condition that $S_{i} \otimes_{R} S_{j} \cong S_{j} \otimes_{R} S_{i}$ is relaxed.

Received 15 August 1984.

1980 Mathematics Subject Classification 17B35.

J. London Math. Soc. (2) 32 (1985) 411-418 
In Section 3, we apply the results from the previous section to the ring $D_{\lambda}$. Briefly the idea is as follows. Let $G / B$ denote the flag variety of the connected semi-simple algebraic group $G$ associated with $g$. Denote by $\mathscr{D}_{\lambda}$ the sheaf of twisted differential operators on $G / B$ constructed in [2]. By translating the large Bruhat cell and taking local sections of $\mathscr{D}_{\lambda}$ we obtain a finite collection of rings $\left\{S_{i}\right\}$ contained between $D_{\lambda}$ and its ring of fractions. Each $S_{i}$ is isomorphic to the $n$-th Weyl algebra (where $n=\operatorname{dim}_{k} n^{+}$) and the construction of $\mathscr{D}_{\lambda}$ ensures that $S_{i} \otimes_{D_{\lambda}} S_{j} \cong S_{j} \otimes_{D_{\lambda}} S_{i}$. When $\lambda$ is dominant regular the equivalence of categories established by Beilinson and Bernstein implies that $S_{1} \oplus \ldots \oplus S_{m}$ is faithfully flat as a right $D_{\lambda}$-module. One may now apply the results of Section 2, together with a result of Joseph and Stafford, to obtain the theorem described above.

We should like to thank Ken Goodearl, Henryk Hecht, Tom Lenagan and Dragan Milicic for their helpful comments.

\section{Weak global dimension and localisation}

For the applications in part 3, it is necessary to consider torsion-theoretic localisation, a slightly more general type of localisation than the usual elementwise Ore localisation. Let $R$ be a (not necessarily commutative) ring. An hereditary torsion class $\mathscr{T}$ for $R$ is a collection of left $R$-modules closed under quotients, submodules, direct sums and extensions. The ring of quotients of $R$ with respect to $\mathscr{T}$ is defined to be $R_{\mathscr{T}}=\lim _{\rightarrow} \operatorname{Hom}_{R}(I, R)$, where the direct limit is taken over the filter of all left ideals $I$ of $R$ such that $R / I$ belongs to $\mathscr{T}$. There is a natural map $\phi: R \rightarrow R_{\mathscr{T}}$, and $R_{\mathscr{T}}$ can then be considered via $\phi$ as a left or right $R$-module. If $R_{\mathscr{T}}$ is flat as a right $R$-module $R_{\mathscr{T}}$ is said to be a perfect left localisation of $R$, and in this situation $\mathscr{T}$ consists precisely of those $R$-modules $M$ such that $R_{\mathscr{T}} \otimes_{R} M=0$. The torsion submodule $\tau(M)$ of an $R$-module $M$ is defined to be the largest submodule of $M$ belonging to $\mathscr{T}$. If $R_{\mathscr{T}}$ is a perfect localisation, then $\tau(M)$ is precisely the kernel of the natural map $\theta: M \rightarrow R_{\mathscr{T}} \otimes_{R} M$. The reader is referred to [18] for a more complete explanation.

If $M$ is an $R$-bimodule its flat dimension as left module is denoted $\operatorname{lfd}_{R}(M)$. When there is no danger of ambiguity or when $M$ is a left $R$-module only, we write $\mathrm{fd}_{R}(M)$.

THEOREM 2.1. Let $R$ be $a$ ring and let $B_{1}, \ldots, B_{n}$ be a finite collection of perfect left localisations of $R$ with associated torsion classes $\mathscr{T}_{1}, \ldots, \mathscr{T}_{n}$, respectively. Suppose that $B_{1} \oplus \ldots \oplus B_{n}$ is faithfully flat as a right $R$-module and that each $\mathscr{T}_{i}$ is closed under $B_{j} \otimes_{R}-$ for $j=1, \ldots, n$. Then

$$
\text { wgldim } R \leqslant \max _{i}\left\{\operatorname{wgldim} B_{i}+\operatorname{lfd}_{R}\left(B_{i}\right)\right\} .
$$

Proof. Clearly we may assume that $\max \left\{\operatorname{wgldim} B_{i}+\operatorname{lfd}_{R}\left(B_{i}\right)\right\}=m<\infty$. For each left $R$-module $M$ let $\eta(M)$ be the number of distinct torsion classes amongst $\mathscr{T}_{1}, \ldots, \mathscr{T}_{n}$ to which $M$ belongs. Suppose the theorem is false, and pick amongst all $R$-modules with flat dimension greater than $m$ a module $M$ such that $\eta(M)$ is greatest possible. Since $B_{1} \oplus \ldots \oplus B_{n}$ is faithfully flat as a right $R$-module, $\mathscr{T}_{1} \cap \ldots \cap \mathscr{T}_{n}=0$, so that $\eta(M)<n$. Choose an index $i$ such that $M$ does not belong to $\mathscr{T}_{i}$ and let $\tau_{i}(M)$ be the torsion submodule of $M$ with respect to $\mathscr{T}_{i}$. Then, if $N=M / \tau_{i}(M)$, there exist exact sequences

$$
0 \longrightarrow \tau_{i}(M) \longrightarrow M \longrightarrow N \longrightarrow 0
$$


and

$$
0 \longrightarrow N \longrightarrow B_{i} \otimes N \longrightarrow B_{i} \otimes N / N \longrightarrow 0
$$

Now, by hypothesis, $B_{i} \otimes N$ belongs to all the torsion classes to which $M$ belongs, and hence so does $B_{i} \otimes N / N$. Because each $B_{i}$ is a perfect left localisation of $R, B_{i} \otimes_{R} B_{i}$ is isomorphic to $B_{i}$ as an $R$ - $R$-bimodule [18, X1.1.2]. The flatness of $B_{i}$ then implies that $B_{i} \otimes\left(B_{i} \otimes N / N\right)=0$. Hence, $\eta\left(B_{i} \otimes N / N\right)>\eta(M)$ and so the choice of $\eta(M)$ implies that $\mathrm{fd}_{R}\left(B_{i} \otimes N / N\right) \leqslant m$. Using the change-of-rings theorem for flat dimension, $\mathrm{fd}_{R}\left(B_{i} \otimes N\right) \leqslant \mathrm{fd}_{B_{i}}\left(B_{i} \otimes N\right)+\operatorname{lfd}_{R}\left(B_{i}\right) \leqslant m$ [4, p. 360, Example 5]. Thus, the exact-sequence lemma for flat dimension implies that $\mathrm{fd}_{R}(N) \leqslant m$.

Similarly, $\tau_{i}(M)$ belongs to $\mathscr{T}_{i}$, so that $\eta\left(\tau_{i}(M)\right)>\eta(M)$. Hence, $\mathrm{fd}_{R}\left(\tau_{i}(M)\right) \leqslant m$ and the top exact sequence implies that $\mathrm{fd}_{R}(M) \leqslant m$, a contradiction.

COROLlaRY 2.2. Let $R, B_{1}, \ldots, B_{n}$ be as in Theorem 2.1. If the $B_{i}$ are also flat as left $R$-modules, then

$$
\text { wgldim } R=\max _{i}\left\{\text { wgldim } B_{i}\right\}=\operatorname{wgldim}\left(B_{1} \oplus \ldots \oplus B_{n}\right) .
$$

The form in which we shall use this result in part 3 is as follows.

COROLlaRY 2.3. Let $R$ be a two-sided Noetherian prime ring with simple quotient ring $Q$. Let $B_{1}, \ldots, B_{n}$ be rings lying between $R$ and $Q$ such that $B_{1} \oplus \ldots \oplus B_{n}$ is $a$ faithfully flat right $R$-module. Suppose further that $B_{i} \otimes_{R} B_{j} \cong B_{j} \otimes_{R} B_{i}$ as $R-R$ bimodules for all $i$ and $j$. Then

$$
\operatorname{gldim} R \leqslant \max _{i}\left\{\operatorname{lgldim} B_{i}+\operatorname{lfd}_{R}\left(B_{i}\right)\right\} .
$$

Proof. Each $B_{i}$ lies between $R$ and $Q$ and is flat as a right $R$-module. Since $Q$ is a right Ore localisation of $R, B_{i}$ must be a perfect left localisation of $R$ by [18, X1.3.4]. Also, each $B_{i}$ is left Noetherian [18, X1.3.9], so that $\operatorname{lgldim} B_{i}=\operatorname{wgldim} B_{i}$. Of course, since $R$ is Noetherian on both the left and right, $\operatorname{gldim} R=\operatorname{lgldim} R=\operatorname{wgldim} R$.

Hence, by Theorem 2.1 , it suffices to check that the torsion class $\mathscr{T}_{i}$ associated with the localisation $B_{i}$ is closed under $B_{j} \otimes_{R}-$ for each $j=1, \ldots, n$. Suppose that $M$ belongs to $\mathscr{T}_{i}$. Then $B_{i} \otimes_{R} B_{j} \otimes_{R} M \cong B_{j} \otimes_{R} B_{i} \otimes_{R} M=0$. Hence, $B_{j} \otimes_{R} M$ belongs to $\mathscr{T}_{i}$, as required.

The following is a condition which is useful in checking the hypothesis of Corollary 2.3. It will be used in the next section.

Proposition 2.4. Let $R$ be a prime Noetherian ring and let $S_{1}$ and $S_{2}$ be two perfect left localisations of $R$. Suppose that each $S_{i}$ is generated as a left and as a right $R$-module by a subset $C_{i}$ and that the elements of $C_{1}$ commute with those of $C_{2}$. Then $S_{1} \otimes_{R} S_{2} \cong S_{2} \otimes_{R} S_{1}$ as $R$ - $R$-bimodules.

Proof. Let $\mathscr{F}_{i}$ be the Gabriel topology associated with the localisation $S_{i}$ of $R$, that is, the set of all left ideals $I$ of $R$ such that $S_{i} \otimes(R / I)=0$. If $Q$ is the classical quotient ring of $R$ then $S_{i}$ can be identified as the set $\left\{x \in Q: I x \subseteq R\right.$ for some $\left.I \in \mathscr{F}_{i}\right\}$. Let $B$ be the subring of $Q$ generated by $S_{1}$ and $S_{2}$. If suffices to show that $S_{1} \otimes_{R} S_{2} \cong B$ as $R$ - $R$-bimodules. 
The flatness of $Q$ as a left $R$-module implies that $S_{1} \otimes_{R} Q$ embeds in $Q \otimes_{R} Q$, which is naturally isomorphic to $Q$. The composite embedding sends $s \otimes q$ to $s q$. Further, the flatness of $S_{1}$ as a right $R$-module implies that $S_{1} \otimes_{R} S_{2}$ embeds naturally in $S_{1} \otimes_{R} Q$. Composing with the above map gives an isomorphism of $R$ - $R$-bimodules from $S_{1} \otimes_{R} S_{2}$ to $S_{1} S_{2}$. But since $C_{1}$ and $C_{2}$ commute and $S_{i}=R C_{i}=C_{i} R$, it is clear that $B=S_{1} S_{2}$. Hence we have an $R$-R-bimodule isomorphism from $S_{1} \otimes_{R} S_{2}$ to $B$.

By analogy with Roos's result [14], there are two directions in which one might hope to weaken the hypotheses of Theorem 2.1. First, one might conjecture that the result is true for infinite sets of localisations $\left\{B_{i}\right\}_{i \in I}$, rather than just finite collections. Secondly, one might think that the conditions on the torsion-classes can be omitted completely. The former is an open question but the latter is false, as the following example shows.

EXAMPLE 2.5. Let $k$ be an algebraically closed field of characteristic zero and let $A$ be the Weyl algebra $k[p, q]$, where $p q-q p=1$. Let $R$ be the subalgebra of $A$ generated by the elements $q, q p, p q p$. Then $R$ is isomorphic to $U(\mathrm{sl}(2, k)) /(\Omega+1)$, where $\Omega$ is the Casimir element [16]. Now [16, Corollary 1] states that the sets $k[q]-\{0\}$ and $k[p q p]-\{0\}$ are left and right Ore subsets of $R$, and that if $S_{1}$ and $S_{2}$ are the respective localisations, then $S_{1} \oplus S_{2}$ is faithfully flat as both a right and left $R$-module. However, both $S_{1}$ and $S_{2}$ are isomorphic to the ring $k(q)[p]$ which has global dimension one, whilst $R$ has infinite global dimension [17]. Of course, in this case, the two Ore sets do not commute and $S_{1} \otimes_{R} S_{2} ¥ S_{2} \otimes_{R} S_{1}$.

\section{Global dimension of primitive factors of $U(\mathrm{~g})$}

Let $G$ be a connected semi-simple algebraic group over an algebraically closed field $k$ of characteristic zero. Fix a Borel subgroup $B$ and a maximal torus $H$ inside $B$, let $W$ be the Weyl group, put $X=G / B$ and let $\operatorname{dim} X=n$. Let $\mathfrak{g}, \mathfrak{b}, \mathfrak{h}$ be the Lie algebras of $G, B, H$, respectively and put $U=U(\mathrm{~g})$, the enveloping algebra of $\mathrm{g}$. Let $R$ be the root system in $\mathfrak{h}^{*}$ with positive roots $R^{+}$and let $\rho$ be the half-sum of the positive roots. For $\alpha \in R$, let $s_{\alpha}$ be the corresponding reflection. A weight $\lambda$ is called dominant if $\lambda\left(h_{y}\right) \notin\{-1,-2,-3, \ldots\}$ for any $\gamma \in R^{+}$. A weight $\lambda$ in $\mathfrak{h}^{*}$ is regular if $\{w \in W \mid w \lambda=\lambda\}=\{e\}$.

For each $\lambda$ in $\mathfrak{h}^{*}$, Bernstein and Beilinson construct a sheaf $\mathscr{D}_{\lambda}$ of twisted differential operators on $X$. The construction is briefly as follows. Let $\mathcal{O}$ be the sheaf of regular functions on $X$. Form the sheaf of algebras $U^{\circ}=\mathcal{O} \otimes_{k} U$ with multiplication given by $(f \otimes Z)(g \otimes Y)=f Z(g) \otimes Y+f g \otimes Z Y$ for $f, g \in \mathcal{O}, Z, Y \in \mathfrak{g}$. Since $g$ acts as global vector fields on $X$ there is a map $\alpha: \mathcal{O} \otimes \mathrm{g} \rightarrow \operatorname{Der} \mathcal{O}$, where $\operatorname{Der} \mathcal{O}$ is the sheaf of $k$-linear derivations. The kernel of $\alpha$ is denoted $b^{\circ}$ and there is a surjection $\mathfrak{b}^{\circ} \rightarrow \mathcal{O} \otimes \mathfrak{h}$. Thus, each $\lambda$ in $\mathfrak{h}^{*}$ induces a map $\lambda^{\circ}: \mathfrak{b}^{\circ} \rightarrow \mathcal{O}$. Let $\mathscr{I}_{\lambda}$ denote the sheaf of ideals of $\mathcal{O} \otimes U$ generated by $\xi-(\lambda-\rho)^{\circ}(\xi)$ for $\xi \in \mathfrak{b}^{\circ}$. Then $\mathscr{D}_{\lambda}$ is defined to be $\mathcal{O} \otimes U / \mathscr{I}_{\lambda}$. For $\lambda$ in $\mathfrak{h}^{*}$, let $M(\lambda)$ be the Verma-module as defined in $[6,17.1]$. Define $D_{\lambda}$ to be $U(\mathrm{~g}) / \mathrm{ann}_{U} M(\lambda)$.

\section{THEOREM 3.1 [2]. (1) $\Gamma\left(X, \mathscr{D}_{\lambda}\right)=D_{\lambda}$;}

(2) If $\lambda$ is dominant and regular there is an equivalence of categories between the category of left $D_{\lambda}$-modules and the category of quasi-coherent left $\mathscr{D}_{\lambda}$-modules given by the mutually inverse functors $\mathscr{M} \rightarrow \Gamma(X, \mathscr{M})$ and $M \rightarrow \mathscr{D}_{\lambda} \otimes_{D_{\lambda}} M$. 
COROLlaRY 3.2. Suppose that $\lambda$ is dominant and regular.

(i) If $U$ is an affine open subset of $X$, then $\Gamma\left(U, \mathscr{D}_{\lambda}\right)$ is flat as a right $D_{\lambda}$-module.

(ii) Let $V_{1}, \ldots, V_{n}$ be an affine open cover of $X$ and let $S_{i}=\Gamma\left(V_{i}, \mathscr{D}_{\lambda}\right)$. Then $\oplus_{i-1}^{n} S_{i}$ is faithfullv flat as a right $D_{\lambda}$-module.

Proof. A left $\mathscr{D}_{\lambda}$-module is quasi-coherent if and only if it is quasi-coherent as an $\mathcal{V}$-module. The corollary then follows from the vanishing of cohomology on affine algebraic varieties. The details may be found in [8].

Proposition 3.3. Let $V$ be an affine open subset of $X$ and let $\lambda$ be dominant regular. Then $\Gamma\left(V, \mathscr{D}_{\lambda}\right)$ is contained between $D_{\lambda}$ and its quotient division ring.

Proof. Let $S=\Gamma\left(V, \mathscr{D}_{\lambda}\right)$. Since $V$ is affine, the maps between $S$ and the local rings at points are just Ore localisations. Hence the quasi-coherence of $\mathscr{D}_{\lambda}$ implies that the rings of local sections on affine open subsets of $X$ can be viewed as subrings of a common quotient division ring, say $E$. Define $\mathscr{E}$ to be the constant sheaf whose module of local sections at any affine open set is just $E$. The module of global sections is then clearly also $E$. By Theorem $3.1, \mathscr{E} \cong E \otimes_{D_{\lambda}} \mathscr{D}_{\lambda}$.

Now since $V$ is affine there is an equivalence of categories between $S$-modules and $\left.\mathscr{D}_{\lambda}\right|_{V}$-modules, given by the mutually inverse functors $\Gamma(V,-)$ and $-\otimes_{S}\left(\left.\mathscr{D}_{\lambda}\right|_{V}\right)$. Since $\left.\mathscr{D}\right|_{V} \cong S \otimes_{S}\left(\left.\mathscr{D}_{\lambda}\right|_{V}\right)$, it is clear that $\left.\mathscr{E}\right|_{V} \cong E \otimes_{D_{\lambda}} S \otimes_{S}\left(\left.\mathscr{D}_{\lambda}\right|_{V}\right)$. But equally clearly (from the definition), $\Gamma(V, \mathscr{E})=E$. Thus

$$
E=\Gamma\left(V, E \otimes_{D_{\lambda}} S \otimes_{S}\left(\left.\mathscr{D}_{\lambda}\right|_{V}\right)\right) \cong E \otimes_{D_{\lambda}} S .
$$

Tensoring on the right over $S$ with $E$ yields that $E \cong E \otimes_{S} E \cong E \otimes_{D_{\lambda}} E$. This implies that the embedding of $D_{\lambda}$ in $E$ must be that of $D_{\lambda}$ into its quotient division ring.

CoROllaRY 3.4. Let $V$ be an open affine subset of $X$ and let $S=\Gamma\left(V, \mathscr{D}_{\lambda}\right)$. Then if $\lambda$ is dominant regular, $S$ is a perfect left localisation of $D_{\lambda}$.

Proof. By Corollary 3.2, $S$ is flat as a right $D_{\lambda}$-module, and by Proposition 3.3, $S$ is contained between $D_{\lambda}$ and its quotient ring. The result then follows from $[18, \mathrm{X} 1.2 .4]$.

THEOREM 3.5. Let $g_{1}, \ldots, g_{l}$ be representatives in $G$ of the Weyl group. Let $V$ be the large Bruhat cell in $X$ and let $V_{i}=g_{i} V$. If $\lambda \in \mathfrak{h}^{*}$ is dominant and regular, then

$$
\operatorname{gldim} D_{\lambda} \leqslant \operatorname{dim} X+\max \left\{\operatorname{lfd}_{D_{\lambda}} \Gamma\left(V_{i}, \mathscr{D}_{\lambda}\right)\right\} .
$$

Proof. Let $S_{i}=\Gamma\left(V_{i}, \mathscr{D}_{\lambda}\right)$ and let $n=\operatorname{dim} X$. Now the $V_{i}$ are translates of $V$, and so must be isomorphic to affine space of dimension $n$. Furthermore, it follows from the definition of $\mathscr{D}_{\lambda}$ that the ring of local sections on $V_{i}$ is just the ring of untwisted differential operators on $V_{i}$. Hence, $S_{i}$ is isomorphic to $A_{n}$, the $n$-th Weyl algebra. Thus, by [14], gldim $S_{i}=\operatorname{gldim} A_{n}=n$. Since $\lambda$ is dominant and regular, Corollary 3.4 and Proposition 3.3 imply that $S_{i}$ is a perfect left localisation of $D_{\lambda}$. Moreover, it follows from the definition of $D_{\lambda}$ that $S_{i}$ is generated as a left and right $D_{\lambda}$-module by $C_{i}=\Gamma\left(V_{i}, \mathcal{O}\right)$. Since $\left\{g_{1}, \ldots, g_{l}\right\}$ is a full set of representatives of the 
Weyl group, the $V_{i}$ form an open affine cover for $X$. Hence, noting that the $C_{i}$ commute, the theorem follows from Corollary 2.3 and Proposition 2.4.

In order to obtain a more precise bound on the global dimension, we first reduce the problem to that of computing $\operatorname{lfd}_{D_{\lambda}} \Gamma\left(V, \mathscr{D}_{\lambda}\right)$. The following lemma will be used twice. Its proof is straightforward and is left to the reader. We denote the right flat dimension of an $R$ - $R$-bimodule $M$ by $\operatorname{~ffd}_{R} M$.

Lemma 3.6. Let $R, S_{1}, S_{2}$ be rings and, for $i=1,2$, let $\phi_{i}: R \rightarrow S_{i}$ be monomorphisms. If $\alpha: S_{1} \rightarrow S_{2}$ and $\beta: R \rightarrow R$ are ring isomorphisms such that $\alpha \phi_{1}=\phi_{2} \beta$, then $\operatorname{lfd}_{R} S_{1}=\operatorname{lfd}_{R} S_{2}$ and $\operatorname{rfd}_{R} S_{1}=\operatorname{rfd}_{R} S_{2}$.

LEMMA 3.7. For any open set $U$ in $X$ and any $g \in G$,

$$
\operatorname{lfd}_{D_{\lambda}} \Gamma\left(U, \mathscr{D}_{\lambda}\right)=\operatorname{lfd}_{D_{\lambda}} \Gamma\left(g U, \mathscr{D}_{\lambda}\right)
$$

Proof. The map $l_{g}: X \rightarrow X$ defined by $x \rightarrow g x$ is a morphism of algebraic varieties and therefore induces a map $\left(l_{g}\right)_{*}: \mathcal{O} \rightarrow \mathcal{O}$ such that $\left(l_{g}\right)_{*}(\Gamma(U, \mathcal{O}))=\Gamma\left(g^{-1} U, \mathcal{O}\right)$. Extend $\left(l_{g}\right)_{*}$ to a map $\pi_{g}: U^{\circ} \rightarrow U^{\circ}$ by letting $\pi_{g}$ act via $\operatorname{Ad}(g)$ on elements of $U(\mathrm{~g})$. Then $\pi_{\theta}$ preserves the multiplication described above, so $\pi_{g}$ is a well-defined isomorphism of sheaves of algebras. Let $\rho_{g}: \operatorname{Der} \mathcal{O} \rightarrow \operatorname{Der} \mathcal{O}$ be the induced map on the tangent sheaf, and let $\alpha: \mathcal{O} \otimes \mathrm{g} \rightarrow$ Der $\mathcal{O}$ be the natural map described above. Then it follows from the definition that $\alpha \pi_{g}=\rho_{g} \alpha$ and hence that $b^{\circ}=\operatorname{ker} \alpha$ is invariant under $\pi_{g}$. Moreover, since $\lambda^{\circ}: \mathfrak{b}^{\circ} \rightarrow \mathcal{O}$ is a fibre map on the quotient $\mathcal{O} \otimes \mathfrak{h}$, for any $\xi \in \mathfrak{b}^{\circ}$, we have that $\pi_{g} \lambda^{\circ}(\xi)=\lambda^{\circ} \pi_{g}(\xi)$. Hence the ideals $\mathscr{I}_{\lambda}=\left(\xi-(\lambda-\rho)^{\circ}(\xi)\right) U^{\circ}$ are invariant under $\pi_{g}$. Thus, for each $\lambda \in \mathfrak{h}^{*}, \pi_{g}$ induces a map $\pi_{g, \lambda}: D_{\lambda} \rightarrow D_{\lambda}$ sending $\Gamma\left(U, \mathscr{D}_{\lambda}\right)$ to $\Gamma\left(g^{-1} U, \mathscr{D}_{\lambda}\right)$ and inducing an automorphism on the global sections $D_{\lambda}$. The result then follows from Lemma 3.6.

Definition 3.8. For $\lambda \in \mathfrak{h}^{*}$, write $R_{\lambda}$ for the root system $R_{\lambda}=\left\{\alpha \in R: \alpha^{*}(\lambda) \in \mathbb{Z}\right\}$, and $W_{\lambda}$ for the Weyl group of $R_{\lambda}$. Set $R_{\lambda}^{\dagger}=R_{\lambda} \cap R^{+}$. Let $\Delta_{\lambda}$ be the corresponding set of simple roots and $S_{\lambda}$ the set of reflections corresponding to $\Delta_{\lambda}$. Let $l_{\lambda}: W_{\lambda} \rightarrow \mathbb{Z}^{+}$be the corresponding length function. Define $n: \mathfrak{h}^{*} \rightarrow \mathbb{Z}^{+}$by $n(\lambda)=\max \left\{l_{\lambda}(w): w \in W_{\lambda}\right\}$. (See [9] for further details.)

A few remarks on $n(\lambda)$ may be made here. First, $n(\lambda)$ satisfies $0 \leqslant n(\lambda) \leqslant \operatorname{dim} X$ for all $\lambda \in \mathfrak{h}^{*}$. If both $\lambda$ and $-\lambda$ are dominant regular (that is, for all $\alpha \in R, \alpha^{*}(\lambda) \notin \mathbb{Z}$ ), then $n(\lambda)=0$, whilst if $\lambda$ is dominant regular and integral (that is, $\alpha^{*}(\lambda) \in \mathbb{N}$ for all $\alpha \in R)$, then $n(\lambda)=\operatorname{dim} X$.

Secondly, for all $w \in W$, a routine calculation reveals that $W_{w \lambda}=w W_{\lambda} w^{-1}$ and $R_{w \lambda}=w R_{\lambda} w^{-1}$. Thus, $n(w \lambda)=n(\lambda)$ and hence $n(\lambda)$ depends only on the Weyl group orbit of $\lambda$.

THEOREM 3.9. If $\lambda$ is regular, then gldim $D_{\lambda} \leqslant \operatorname{dim} X+n(\lambda)$.

Proof. For $\lambda$ regular, there exists $w \in W$ such that $w \lambda$ is dominant regular. Since $D_{w \lambda} \cong D_{\lambda}$ and $n(w \lambda)=n(\lambda)$, we may therefore assume that $\lambda$ is dominant. Let $V$ be the large Bruhat cell as before. Then, by Theorem 3.5 and Lemma 3.7, it suffices to show that $\operatorname{lfd}_{D_{\lambda}} \Gamma\left(V, \mathscr{D}_{\lambda}\right) \leqslant n(\lambda)$. Since by $\left[1\right.$, p. 44] $\mathscr{D}_{\lambda}$ is isomorphic to $\mathscr{D}_{-\lambda}^{0}$, the opposite sheaf of $\mathscr{D}_{-\lambda}$, this is equivalent to showing that $\operatorname{rfd}_{D_{-\lambda}} \Gamma\left(V, \mathscr{D}_{-\lambda}\right) \leqslant n(\lambda)$. 
Now let $w_{0}$ be the longest element of the Weyl group, let $\mu=w_{0} \lambda$ and let $i: D_{\mu} \rightarrow A_{n}$ be the embedding, described by Conze [5], of $D_{-w_{0} \lambda}$ into the $n$-th Weyl algebra. Let $j: D_{-\lambda} \rightarrow \Gamma\left(V, \mathscr{D}_{-\lambda}\right)$ be the restriction map. Then in [8] it is shown that there exist isomorphisms $\tau: D_{-\lambda} \rightarrow D_{\mu}$ and $\psi: \Gamma\left(V, \mathscr{D}_{-\lambda}\right) \rightarrow A_{n}$ such that $i \tau=\psi j$. But then it follows from Lemma 3.6 that

$$
\operatorname{rfd}_{D_{\mu}}\left(A_{n}\right)=\operatorname{rfd}_{D_{-\lambda}}\left(\Gamma\left(V, \mathscr{D}_{-\lambda}\right)\right) .
$$

Now, in [11, Theorem 5.8] it is shown that $\operatorname{rfd}_{D_{\mu}}\left(A_{n}\right) \leqslant n(\mu)$. Moreover, $n(\mu)=n\left(-w_{0} \lambda\right)=n\left(w_{0} \lambda\right)=n(\lambda)$. Hence, $\operatorname{rfd}_{D_{-\lambda}}\left(\Gamma\left(V, \mathscr{D}_{-\lambda}\right)\right) \leqslant n(\lambda)$, as required.

Corollary 3.10. If $\lambda$ is regular, then $\operatorname{gldim} D_{\lambda}=\operatorname{dim} X+n(\lambda)$.

Proof. Let $n=\operatorname{dim} X$. As above, we may assume that $\lambda$ is antidominant. It is shown in [12] that inj $\operatorname{dim}\left(D_{\lambda}\right)=2 n-\min _{w \in W}\{d(L(w \lambda))\}$, where $L(w \lambda)$ is the unique simple factor of the Verma module $M(w \lambda)$ and $d(-)$ denotes the Gelfand-Kirillov dimension. If $w_{0}$ is the longest element of the Weyl group, then certainly $n(\lambda)=l_{\lambda}\left(w_{0}\right)$. On the other hand, it is shown in $[10, \S 8.18]$ that (for $\lambda$ antidominant and regular), $d\left(L\left(w_{0} \lambda\right)\right)=n-l_{\lambda}\left(w_{0}\right)$. Hence

$$
n+n(\lambda)=n+l_{\lambda}\left(w_{0}\right)=2 n-d\left(L\left(w_{0} \lambda\right)\right) \leqslant \operatorname{inj} \operatorname{dim}\left(D_{\lambda}\right) .
$$

Thus gldim $D_{\lambda}=n+n(\lambda)$, as required.

Note. Bernstein has recently announced that Theorem 3.9 also follows from a more general theorem on the vanishing of cohomology (similar to that in [2]) together with a spectral sequence of Grothendieck.

\section{References}

1. A. Beilinson and I. N. Bernstein, 'A generalisation of Casselman's submodule theorem', Representation theory of Lie groups (Birkhauser, Boston 1983).

2. A. Beilinson and I. N. Bernstein, 'Localisation de g-modules', C.R. Acad. Sci. Paris Sér. I 292 (1981) 15-18.

3. J.-E. BJORK, 'The global homological dimension of some algebras of differential operators', Invent. Math. 17 (1972) 67-78.

4. H. Cartan and S. EILenberg, Homological algebra (University Press, Princeton 1956).

5. N. CONZE, 'Algèbres d'operateurs différentiels et quotients des algèbres enveloppantes', Bull. Soc. Math. France 102 (1974) 379-415.

6. J. DiXmieR, Enveloping algebras (North-Holland, Amsterdam 1977).

7. K. R. GoodeaRL, 'Global dimension of differential operator rings', Trans. Amer. Math. Soc. 209(1975) 65-85.

8. T. J. Hodges and S. P. SMITH, 'Differential operators on the flag variety and the Conze embedding', preprint, University of Warwick 1984.

9. J. C. JANTZEN, Moduln mit einem hochsten Gewicht, Lecture Notes in Mathematics 750 (Springer, Berlin 1979).

10. A. JosEPH, 'On the variety of a highest weight module, J. Algebra 88 (1984) 238-278.

11. A. JoSEPH and J. T. STAFFORD, 'Modules of $k$-finite vectors over semi-simple Lie algebras,'Proc. London Math. Soc. (3) 49 (1984) 361-384.

12. T. Levasseur, 'Dimension injective des quotients primitifs minimaux de l'algèbre enveloppante d'une algèbre de Lie semi-simple', C.R. Acad. Sci. Paris Sér. I 292 (1981) 385-387.

13. J. C. MCCONNELL, 'On the global dimension of some rings,' Math. Z. 153 (1977) 253-265.

14. J.-E. Roos, 'Détermination de la dimension homologique globale des algèbres de Weyl', C.R. Acad. Sci. Paris Sér. A. 274 (1972) 23-26.

15. J.-E. Roos, 'Propriétés homologiques des quotients primitifs des algèbres enveloppantes des algèbres de Lie semi-simple', C.R. Acad. Sci. Paris Sér. A. 276 (1973) 351-354. 
16. J.-E. Roos, 'Compléments à l'étude des quotients primitifs des algèbres enveloppantes des algèbres de Lie semi-simple', C.R. Acad. Sci. Paris Sér. A. 276 (1973) 447-450.

17. J. T. StafFord, 'Homological properties of the enveloping algebra $U\left(\mathrm{sl}_{2}\right)$ ', Math. Proc. Cambridge Philos. Soc. 91 (1982) 29-37.

18. B. StEnStrom, Rings of quotients (Springer, New York 1975).

Department of Mathematics University of Utah

Salt Lake City

Utah 84112

USA

Current address:

Department of Mathematics

Mail Location 25

University of Cincinnati

Cincinnati

Ohio 45221

USA
Department of Mathematics University of Warwick Coventry CV4 7AL 